\title{
Cortex- and striatum- derived neural stem cells produce distinct progeny in the olfactory bulb and striatum
}

\author{
Sandrine Willaime-Morawek ${ }^{*}$ and Derek van der Kooy \\ Department of Molecular Genetics, University of Toronto, CCBR, Toronto, ON, Canada
}

Keywords: astrocyte, cell differentiation, cell migration, Mus musculus, neuron

\begin{abstract}
Neural stem cells can be isolated from the mouse embryonic cortex but do not persist in the adult cortex. In contrast, neural stem cells from the striatal embryonic germinal zone persist in the adult subependyma. Emx1-lineage analysis revealed that cortex-derived neural stem cells survive and migrate ventrally into the subependyma where they intermix with the host striatal neural stem cells [S. Willaime-Morawek et al. (2006) J. Cell Biol. 175, 159-168]. Cortex-derived cells proliferate faster in the subependyma and reach the olfactory bulb earlier than striatum-derived cells. In the olfactory bulb, cortex-derived cells produce more cells and more dopaminergic neurons in the glomerular layer than striatum-derived cells. Cortex-derived cells also give rise to more astrocytes and less neurons in the striatum than striatum-derived cells. Thus, history matters; cortex-derived neural stem cells in the subependyma give rise to progeny in the olfactory bulb and striatum but in different proportions than striatum-derived neural stem cells.
\end{abstract}

\section{Introduction}

Neural stem cells have been isolated from the embryonic cerebral cortical germinal zone (Davis \& Temple, 1994) but do not appear to persist in the adult cortex (Laywell et al., 2000). In contrast, stem cells from the striatal germinal zone persist into adulthood in the subependyma, the adult striatal germinal zone that surrounds the forebrain lateral ventricles (Kornack \& Rakic, 2001; Seaberg et al., 2005). Given that stem cells are defined by their long-term selfrenewal capacity, embryonic cerebral cortical neural stem cells should persist if these cells are really stem cells. We sought to determine their fate by using a region-specific molecular marker, the Emxl gene, to express the Cre recombinase. Emxl is a homeobox gene expressed in a dorsal-restricted cortical pattern in the developing forebrain (Simeone et al., 1992) that persists in many adult cortical cells (Chan et al., 2001). Emx1-IRES-Cre mice, which express Cre recombinase in a spatial and temporal pattern that recapitulates endogenous Emx1 expression (Gorski et al., 2002), were mated to the reporter strain lacZ/enhanced green fluorescent protein (Z/EG), which expresses enhanced green fluorescent protein (GFP) upon Cre-mediated excision (Novak et al., 2000). These Emx1-Z/EG mice revealed that GFPpositive embryonic cortical neural stem cells survive and migrate ventrally into the subependyma (Willaime-Morawek et al., 2006). As the cortex-derived neural stem cells are now intermixed with the host striatal neural stem cells in the adult subependyma, would the disparate history of these two populations of cells make a difference in the properties or fate of these stem cells? We answered this question by analysing the proliferation, migration and phenotype of their progeny.

Correspondence: Dr Sandrine Willaime-Morawek, *present address below.

E-mail: s.willaime-morawek@soton.ac.uk

* Present address: Division of Clinical Neurosciences, University of Southampton, LD72, South Laboratory Block, Southampton General Hospital, Mailpoint 806, Southampton SO16 6YD, UK

Received 1 October 2007, revised 12 February 2008, accepted 13 March 2008

\section{Materials and methods}

Mice

Emx1-IRES-Cre mice were generated and genotyped as described by Gorski et al. (2002). Z/EG mice, which express lacZ prior to Cre excision and GFP after Cre excision (Novak et al., 2000), were a gift from C. Lobe (Sunnybrook and Women's College Health Centre, Toronto). Mice were bred, housed and treated in accordance with the Animal Care Committee of the University of Toronto animal facility. Every effort was made to minimize the numbers of animals utilized in studies. Our animal protocol was reviewed and approved by the University of Toronto Animal Care Committee.

\section{BrdU labelling and immunohistochemical detection}

To examine the proliferation of the subependymal cells, Emx1-Z/EG mice were injected with bromodeoxyuridine (BrdU) (Sigma;

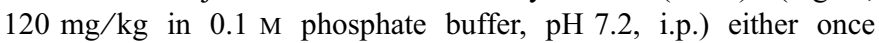
or for a total of five injections every $3 \mathrm{~h}$ and killed $1 \mathrm{~h}$ after the unique or final injection. Animals were killed with avertin overdose and transcardially perfused with $4 \%$ paraformaldehyde and $0.4 \%$ picric acid in $0.16 \mathrm{M}$ phosphate buffer $(\mathrm{pH}$ 6.9). Brains were postfixed overnight in the perfusing solution at $4{ }^{\circ} \mathrm{C}$ and then cryoprotected for at least $24 \mathrm{~h}$ in $30 \%$ sucrose in $0.1 \mathrm{M}$ phosphate buffer $(\mathrm{pH} 7.2)$.

Serial $15 \mu \mathrm{m}$ coronal cryosections were directly mounted onto Superfrost Plus slides (Fisher Scientific). Sections were initially treated with $4 \mathrm{M} \mathrm{HCl}$ for $30 \mathrm{~min}$ to denature cellular DNA. Rat antiBrdU (Abcam; 1 : 100) and fluorescein isothiocyanate anti-rat (Jackson; 1 : 200) antibodies were used to detect BrdU. Three to six $15 \mu \mathrm{m}$ coronal sections of the desired regions [antero-posterior levels for: lateral ventricle, Bregma 0 to +1 ; rostral migratory stream (RMS), Bregma +2 to +2.5 ; olfactory bulb, Bregma +3.5 to +4 ] were picked randomly and analysed per animal. Optical dissector counts were performed to confirm GFP-positive cells counts. The specificity of immunostaining was confirmed by the absence of detectable 


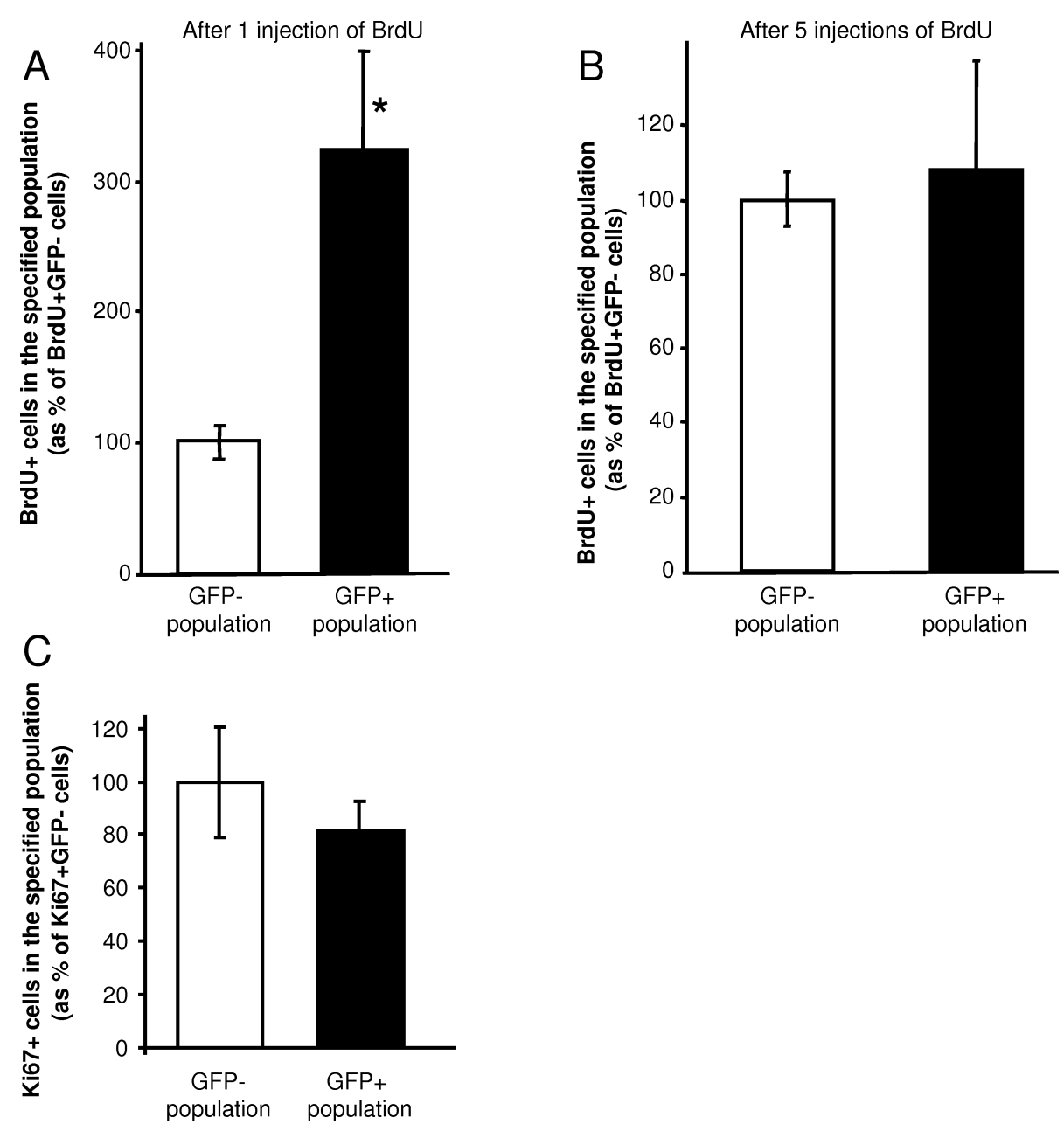

FIG. 1. Emx1-lineage GFP-positive cells proliferate faster than GFP-negative cells in the adult subependyma. Quantification of BrdU- or Ki67-labelled cells in the lateral ventricle subependyma. Adult Emx1-Z/EG mice were injected with BrdU (120 mg/kg, i.p.) once (A) or every $3 \mathrm{~h}$ for a total of five injections (B) and killed $1 \mathrm{~h}$ following the single or last BrdU injection, respectively. All GFP-positive BrdU-positive labelled cells and/or pan-histone-positive cells (total numbers of cells) were counted around the rostral lateral ventricle. (C) All GFP-positive Ki67-positive and/or DAPI-positive cells were counted around the rostral lateral ventricle. The graphs represent the proportions of BrdU-positive or Ki67-positive cells in the specified population (either GFP-positive population or GFPnegative population, evaluated by subtracting GFP-positive cells from the pan-histone-positive or DAPI-positive cells). The data represent $n=4$ independent experiments; 2844 cells (A), 1901 cells (B) and 3937 cells (C) were counted. Error bars represent the SEM. * $t=-2.691, P=0.025$ according to Student's $t$-test.

fluorescence in sections processed identically except for the omission of primary antibodies.

\section{Immunohistochemistry}

Brains were fixed, embedded, cryosectioned into $15 \mu \mathrm{m}$ sections and immunostained using: (i) rabbit polyclonal antibodies: anti-calbindin (Chemicon; $1: 500$ ), anti-calretinin (Sigma; $1: 2000$ ), anti-DARPP32 (Chemicon; $1: 500$ ), anti-GAD65/67 (Chemicon; $1: 200$ ), anti-GFP (Abcam; 1 : 1000), anti-Ki67 (BD Pharmingen; $1: 10$ ), anti-Tbx21 (gift of Y. Yoshihara, RIKEN Brain Research Institute, Saitama, Japan; $1: 1000$ ), anti-tyrosine hydroxylase (Chemicon; $1: 250)$ and (ii) mouse monoclonal antibodies: anti-glial fibrillary acidic protein (Sigma; $1: 400$ ), anti-GFP (Chemicon; $1: 500$ ), antiGAD65 (Chemicon; $1: 250$ ), anti-pan-histone (Abcam; $1: 500$ ), antiNeuN (Chemicon; $1: 200$ ), anti-Pax6 (Developmental Hybridoma Bank; $1: 50)$ and anti-rabbit or anti-mouse Alexa Fluor 488 or 568 (Molecular Probes; $1: 300$ ). The specificity of immunostaining was confirmed by the absence of detectable fluorescence in sections processed identically except for the omission of primary antibodies.
Cell nuclei were counterstained with DAPI. Optical dissector counts were performed to confirm GFP-positive cell counts. Fluorescent images were visualized and captured using an Olympus IX81 Motorized Inverted Research Microscope and captured using Olympus Microsuite Version 3.2 image analysis software (Soft Imaging System Corp.). When reconstructing pseudocoloured pictures, the green channel was used for GFP and the red channel for other markers for the convenience of the reader, regardless of the secondary antibodies used.

\section{Lentivirus injection}

A lentivirus containing a Ds-red reporter gene (from pIRES2-DsRed Express vector, Clontech) was utilized to label subependymal cells in vivo with a non-diluting lineage marker. Briefly, lentivirus was collected from confluent $293 \mathrm{~T}$ cells for $48 \mathrm{~h}$ at $32{ }^{\circ} \mathrm{C}, 0.45 \mu \mathrm{m}$ filtered, spun at $87000 \mathrm{~g}$ for $2 \mathrm{~h}$, aliquotted and stored at $-70{ }^{\circ} \mathrm{C}$. The viral concentrate titre was $10^{8}$ active virions $/ \mathrm{mL}$ as determined by infection of $293 \mathrm{~T}$ cells. Emx1-Z/EG mice (1 day old) were anaesthetized using isoflurane and unilateral injections of $1.0 \mu \mathrm{l}$ of 
lentivirus were made directly into the lateral ventricle. Following treatments, animals were killed with avertin overdose and perfused transcardially with $4 \%$ paraformaldehyde in $0.1 \mathrm{M}$ phosphate buffer $(\mathrm{pH}$ 7.2). Brains were post-fixed in the perfusing solution overnight at
$4{ }^{\circ} \mathrm{C}$ and then cryoprotected for at least $24 \mathrm{~h}$ in $30 \%$ sucrose in $0.1 \mathrm{M}$ phosphate buffer ( $\mathrm{pH}$ 7.2). Serial $15 \mu \mathrm{m}$ coronal cryosections were directly mounted onto Superfrost Plus slides (Fisher Scientific). Every third $15 \mu \mathrm{m}$ coronal section was analysed quantitatively.
A

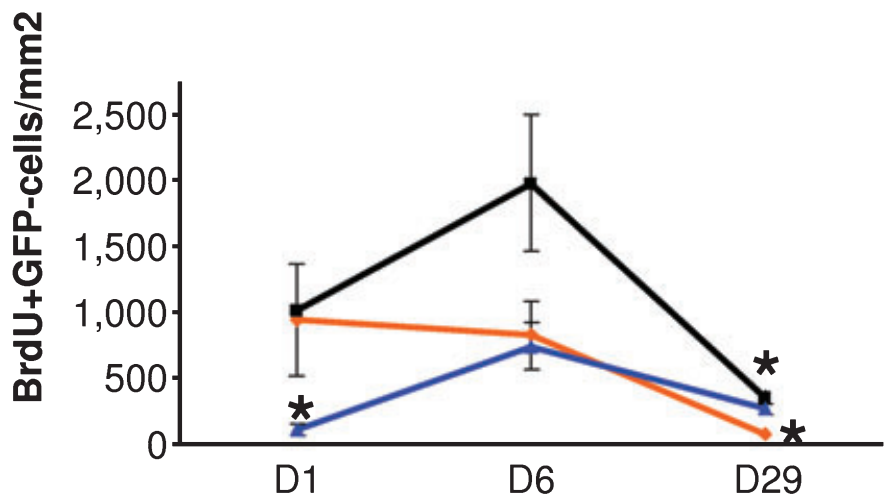

B

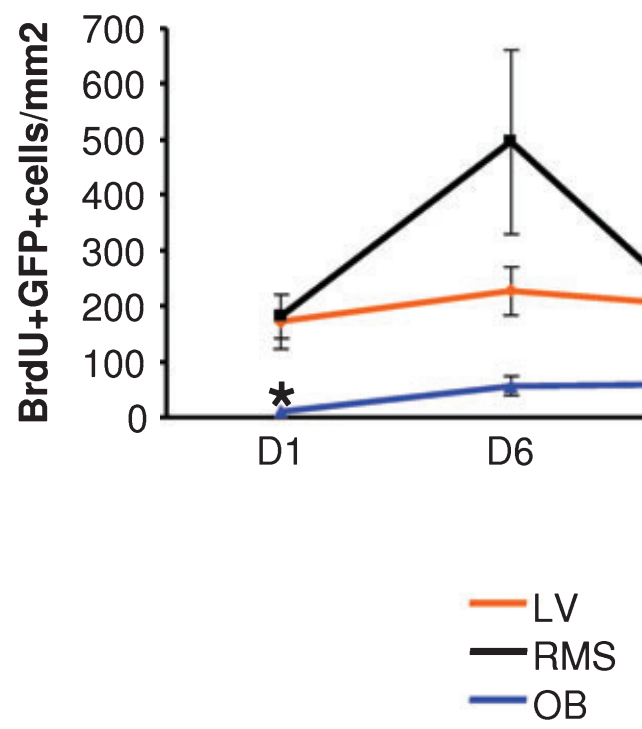

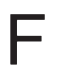
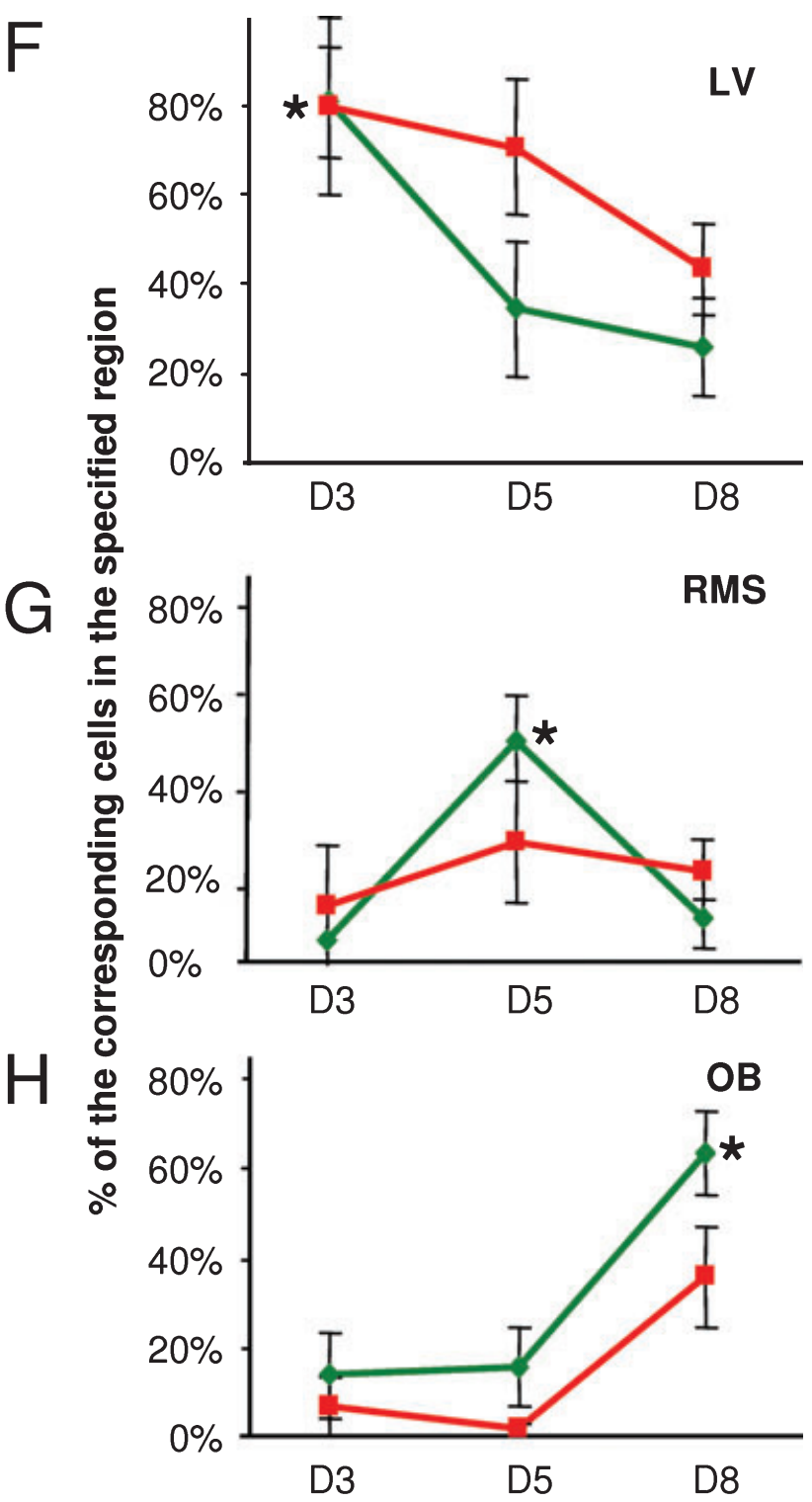

Ds-red+ GFP+ Ds-red+ GFP-
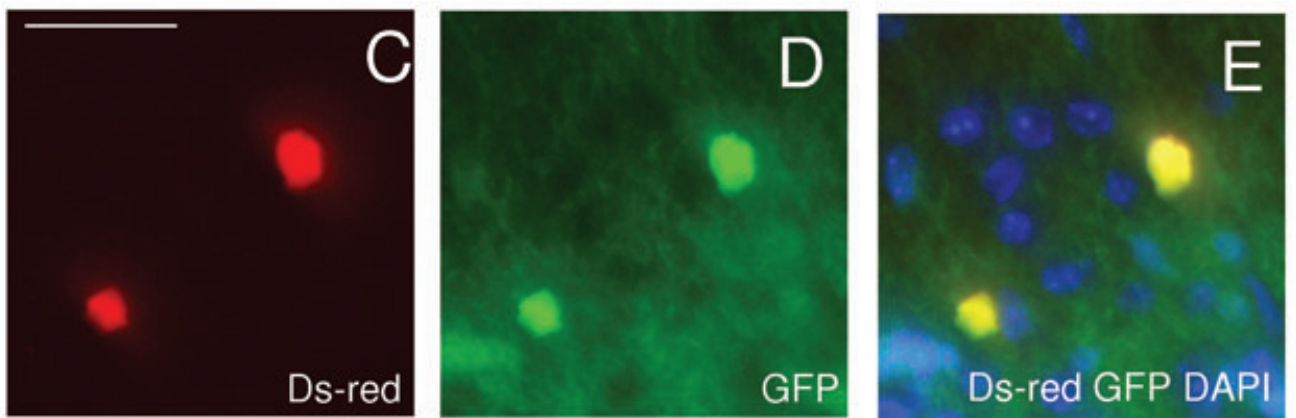


\section{Results}

We have previously shown that embryonic cortical neural stem cells migrate ventrally and persist as post-natal stem cells in the striatal subependymal lining of the lateral ventricle (Willaime-Morawek et al., 2006). As these cells were seemingly completely intermixed with resident striatal stem cells, we asked if the post-natal progeny of these cortex-derived neural stem cells have different fates than the progeny of the host striatal neural stem cells.

In order to analyse proliferation, BrdU was injected into adult Emx1-Z/EG mice. We first performed a single injection of BrdU and killed the mice after $1 \mathrm{~h}$, which allowed labelling of the proliferating cells in S-phase at the time of the injection. BrdU-positive cells and GFP-positive cells were identified using the corresponding antibodies. GFP-negative cells were identified using pan-histone antibody (Fig. 1A and B) or DAPI (Fig. 1C) and checking for absence of the GFP signal. All of the cells in the rostral subependyma were counted, as GFP-positive cells are present in both stem cell and supporting cell populations. A greater percentage of the Emx1-lineage GFP-positive population than of the GFP-negative population were labelled with BrdU in the rostral subependyma (Fig. 1A). This result could be explained by two processes: (i) a higher proportion of proliferating cells in the GFP-positive population than in the GFP-negative population or (ii) the same proportions of proliferating cells in both populations but cells proliferating faster with a shorter cell cycle time in the GFP-positive population, thus more would be in S-phase at the time of the injection. To discriminate between these two possibilities, five BrdU injections were given over the period of one cell cycle time (12.7 h) (Morshead \& van der Kooy, 1992) to label all of the constitutively proliferating subependymal cells (Fig. 1B). The same proportions of proliferating cells were seen in both populations. These results were confirmed using Ki67 to label all of the proliferating cells (Fig. 1C). There are no more GFP-positive cells labelled than GFPnegative cells in the subependyma, presumably because new GFPpositive cells migrate away from the subependyma, stop proliferating or experience cell death. No difference between GFP-positive and GFP-negative populations was observed in cell death, detected by TUNEL, in the rostral subependyma. Thus, we conclude that Emx1lineage GFP-positive cells have a shorter cell cycle time and proliferate faster than GFP-negative cells in the adult subependyma.

To confirm that a fate of the GFP-positive cell progeny was to follow the RMS to the olfactory bulb, as some of the GFP-negative cells do (Willaime-Morawek et al., 2006; Kohwi et al., 2007; Young et al., 2007), we followed over time cells initially in the subependyma labelled with BrdU (Fig. 2A and B). BrdU-positive GFP-positive cells, like BrdU-positive GFP-negative cells, migrated away from the lateral ventricle subependyma through the RMS to the olfactory bulb. As BrdU-positive GFP-negative cells migrated through the RMS, the BrdU-positive GFP-negative cell number decreased in the lateral ventricle and increased in the RMS and core of the olfactory bulb, when comparing days 1 and 6 after the BrdU injections (Fig. 2A). Double-labelled BrdU-positive GFP-positive cell numbers (Fig. 2B) stayed stable in the lateral ventricle from day 1 to day 29 , as migration and/or cell death may compensate for the faster proliferation. These numbers increased in the RMS from day 1 to day 6 , then decreased at day 29 and increased in the core of the olfactory bulb from day 1 until day 29. There is no massive increase in the core of the olfactory bulb at day 29 for both cell populations, as most of the cells may have left this region to differentiate into the granular and glomerular layers of the olfactory bulb at that point. In conclusion, like the GFP-negative cells, the progeny of cortex-derived GFPpositive cells in the striatal subependyma migrate to the olfactory bulb through the RMS.

To permanently mark the GFP-positive cells migrating to the olfactory bulb and avoid a dilution effect of the BrdU label due to proliferation, Ds-red lentivirus was injected into the lateral ventricle of Emx1-Z/EG mice. In order to label more GFP-positive cells we employed neonates (post-natal day 1), in which more GFP-positive cells and proliferating germinal zone cells in general are present in the subependyma (Willaime-Morawek et al., 2006). At 8 days after the lentivirus injection, Ds-red-positive GFP-positive cells could be detected in the olfactory bulb (Fig. 2C-E). Cells in the lateral ventricle subependyma, RMS and olfactory bulb were counted at 3, 5 or 8 days after the Ds-red lentivirus injection (Fig. 2F-H). The results show that: (i) infected GFP-positive and GFP-negative cells leave the lateral ventricle after a few days with a higher proportion for GFP-positive cells compared with GFP-negative cells at days 5 and 8 (Fig. 2F); (ii) a higher proportion of GFP-positive than GFP-negative cells reach the RMS after 5 days and (iii) a higher proportion of the Ds-red-positive GFP-positive cells reach the olfactory bulb by day 8 . Although only Ds-red-positive GFP-positive cell counts show significant differences, they follow the same known trend as Ds-red-positive GFP-negative cells. These results show that GFP-positive cells leave the lateral ventricle and reach the RMS and olfactory bulb in higher proportions than do the GFP-negative cells. Given that the absolute numbers of Ds-red-labelled cells are about the same at each time point (day 3, $12.9 \pm 3.3$; day $5,14.8 \pm 4.1$; day $8,15.67 \pm 1.41$ ) and that no difference between GFP-positive and GFP-negative populations was observed in cell death, detected by TUNEL, in the olfactory bulb, it is unlikely that these results are due to differences in survival. Thus, the differences observed could be explained by a faster migration in the RMS and olfactory bulb of the GFP-positive cells compared with GFP-negative cells.

Counting the GFP-positive cells in the different layers of the adult olfactory bulb revealed that $21.3 \pm 1.6 \%$ of the cells in the granular layer and $20.3 \pm 1.5 \%$ of the cells in the glomerular layer are GFPpositive. To assess the final destination of GFP-positive cells arriving from the lateral ventricle into the olfactory bulb, the laminar distributions of BrdU-positive GFP-positive and BrdU-positive GFPnegative cells in the olfactory bulb were analysed. BrdU-positive

FIG. 2. Emx1-lineage GFP-positive cells migrate through the RMS to the olfactory bulb. (A and B) Adult Emx1-Z/EG mice were injected with BrdU every $3 \mathrm{~h}$ over $12 \mathrm{~h}$ and killed as indicated. BrdU-positive and GFP-positive cells were counted in the rostral subependyma of the lateral ventricles, in the RMS and in the core of the olfactory bulb (corresponding to the arrival of the RMS in the olfactory bulb). The data represent $n=4$ independent experiments at each time point; 971 cells were counted at day 1;2396 cells at day 6 and 1184 cells at day 29. Error bars represent the SEM. *Significantly different from the two other time points in the region analysed, by Kruskal-Wallis one-way ANOvA on ranks followed by Dunn's post-hoc test. (A) For lateral ventricle $(\mathrm{LV})$ : $\mathrm{H}(2)=14.561, P<0.001$; for RMS: $\mathrm{H}(2)=12.258, P=0.002$; for OB: $\mathrm{H}(2)=19.081, P<0.001$. (B) For OB: $\mathrm{H}(2)=12.617, P=0.002$. $\mathrm{H}(2)$ is the test statistic for Kruskal-Wallis oneway ANOVA on Ranks with 2 degrees of freedom. (C and E) Olfactory bulb cryosections from post-natal day (P)9 Emx1-Z/EG mice injected with Ds-red lentivirus at P1. These micrographs represent Ds-red lentivirus (C and E, red), GFP (D and E, green) and DAPI (E, blue). Scale bar, $25 \mu \mathrm{m}$. (F-H) Emx1-lineage GFP-positive cells migrate faster through the RMS to the olfactory bulb. Graphs represent percentages of the Ds-red lentivirus-positive and/or GFP-positive cells in the specified region at each time point after Ds-red lentivirus injections into lateral ventricle of P1 Emx1-Z/EG mice killed 3, 5 or 8 days later. The data represent $n=8$ (D3), $n=4$ (D5) and $n=6$ (D8) animals. Numbers represent the mean \pm SEM. *Ds-red-positive GFP-positive time point significantly different from the two other Dsred-positive GFP-positive time points by two-way ANOvA followed by Holm-Sidak's post-hoc test: (F) $P=0.013 ;(\mathrm{G}) P=0.010$; (H) $P=0.001$. OB, core of the olfactory bulb (in A and B) or whole olfactory bulb (in F and H). D1 to D29, day 1 to day 29. 

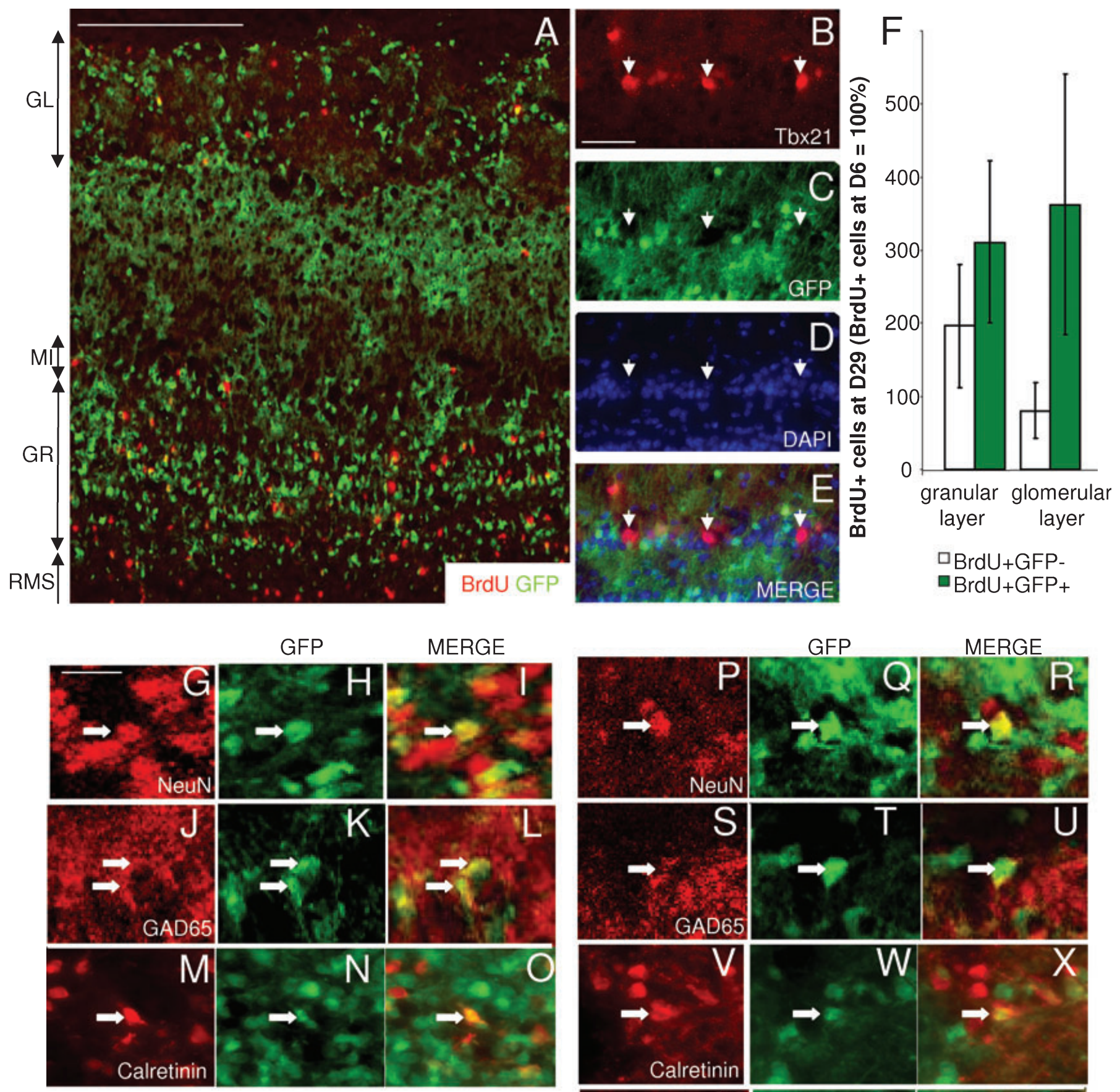

\section{Granular Layer}

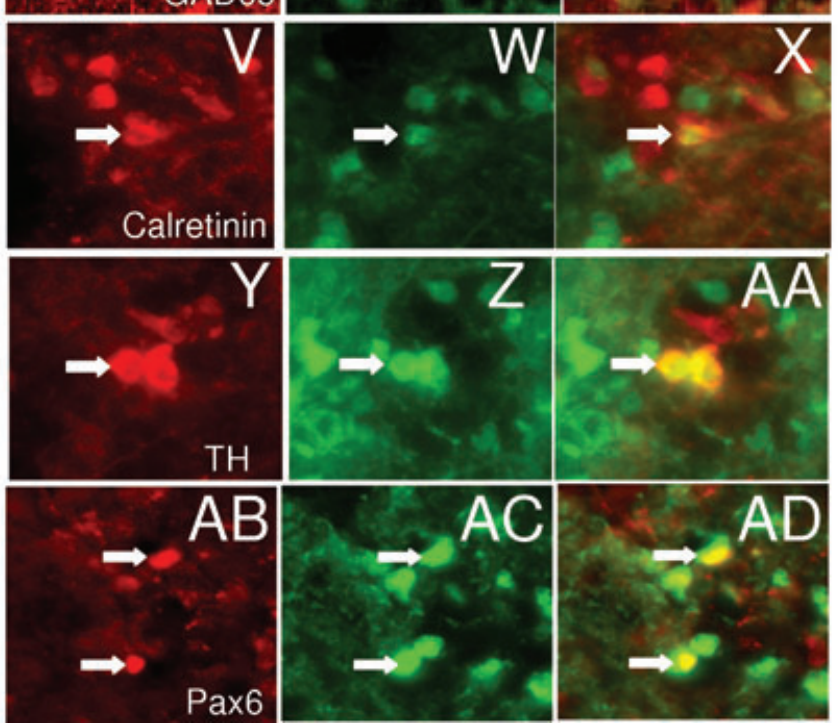


GFP-positive double-labelled cells were found in all three cell layers of the olfactory bulb (granular layer, mitral cell layer and glomerular layer) (Fig. 3A). Because new mitral neurons are not known to be born in the adult olfactory bulb, a mitral cell marker, Tbx21, was used to better characterize the cells in this layer. The GFP-positive cells in the mitral cell layer did not stain for this mitral cell marker (Fig. 3B-E) and thus may rather be displaced granule cells or cells en route to the glomerular layer (Luskin, 1993). The numbers of BrdU-positive cells in the granular and glomerular layers were quantified at 6 and 29 days after the BrdU injections (Fig. 3F). At day 6, $25.9 \pm 5.9$ BrdUpositive GFP-negative cells and 4.5 \pm 1.4 BrdU-positive GFP-positive cells were counted per field (equivalent to $1.3 \mathrm{~mm}^{2}$ ) in the granular layer and 15.0 \pm 4.4 BrdU-positive GFP-negative cells and $1.2 \pm 0.3$ BrdU-positive GFP-positive cells were counted per field in the glomerular layer. At day 29, $51.0 \pm 10.4$ BrdU-positive GFP-negative cells and 14.1 \pm 0.7 BrdU-positive GFP-positive cells were counted per field in the granular layer and $12.1 \pm 2.2$ BrdU-positive GFPnegative cells and 4.2 $\pm 1.0 \mathrm{BrdU}$-positive GFP-positive cells were counted per field in the glomerular layer. In the granular layer, there were two to three times more BrdU-positive GFP-negative cells and BrdU-positive GFP-positive cells at 29 days compared with 6 days after the BrdU injections. In the glomerular layer, there were as many BrdU-positive GFP-negative cells after 29 days as after 6 days. However, there were 3.5 times more BrdU-positive GFP-positive cells found in the glomerular layer at 29 days post-BrdU compared with 6 days post-BrdU. This suggests that GFP-positive cells have a tendency to migrate preferentially to the glomerular layer compared with the granular layer. To better characterize the cells in these two cell layers, GFP-positive cells were double labelled with different interneuron markers. GFP-positive cells, like GFP-negative cells, make GABAergic and calretinin-positive neurons in the granular and glomerular layers, as well as dopaminergic neurons in the glomerular layer (Fig. 3G-AA). These results are in accordance with recent findings using the Emx1-lineage marker (Willaime-Morawek et al., 2006; Kohwi et al., 2007; Young et al., 2007) or labelling dorsal radial glia (Ventura \& Goldman, 2007; Merkle et al., 2007). Some of the GFP-positive cells in the glomerular layer also express Pax6 (Fig. 3, $\mathrm{AB}-\mathrm{AD}$ ), as do some GFP-negative cells in this layer. Quantifying the Pax6-positive cells revealed that $24.1 \pm 5.0 \%$ of the GFP-positive cells are also Pax6-positive, compared with only $8.0 \pm 1.5 \%$ of the GFP-negative cells that are also Pax6-positive $(n=4 ; P=0.022)$. Quantifying the TH-positive cells revealed that $20.2 \pm 2.8 \%$ of the GFP-positive cells are also TH-positive, compared with only $11.9 \pm 1.6 \%$ of the GFP-negative cells that are also TH-positive $(n=6 ; P=0.017)$. Stated in another way, $30.0 \pm 2.0 \%$ of the THpositive cells are GFP-positive. This confirms that Emx1-lineage cells are more likely to give rise to dopaminergic neurons than GFPnegative cells (Kohwi et al., 2007; Merkle et al., 2007), whereas others reported no differences (Ventura \& Goldman, 2007; Young et al., 2007).
We further analysed the sections through the forebrain of adult BrdU-injected Emx1-Z/EG mice and found some sparsely distributed GFP-positive cells in the post-mitotic striatum, which represent $0.5 \%$ of the cells in the adult striatum (counting DAPI-positive and GFPpositive cells in striatum slices with $19.8 \pm 2.1$ GFP-positive cells $/ \mathrm{mm}^{2}$ ). Most of these cells were found in the dorso-lateral and dorso-medial quarters of the striatum. When analysis was performed after five injections of BrdU in adult mice, some of these GFP-positive cells were also BrdU-positive (Fig. 4A-C), which means that they proliferated in the adult brain.

To determine the phenotypes of the GFP-positive cells in the striatum, staining with astrocytic (glial fibrillary acidic protein) or neuronal (NeuN) markers was carried out. The GFP-positive population comprised both astrocytes and neurons in the post-mitotic striatum (Fig. 4E-J). GFP-positive astrocytes tended to be close to the corpus callosum, whereas GFP-positive neurons tended to be further spread into the striatal tissue. Quantifying the double-labelled glial fibrillary acidic protein-positive or NeuN-positive and GFP-positive cells revealed that GFP-positive cells are more likely to become astrocytes and less likely to become neurons compared with GFPnegative cells (Fig. 4D). However, even the GFP-positive cells in the post-mitotic striatum made different types of striatal neurons, such as GABAergic, DARPP32-positive, calretinin-positive and calbindinpositive cells (Fig. 4K-V).

\section{Discussion}

Cortex-derived neural stem cells and endogenous striatal neural stem cells that are intimately intermixed in the post-natal striatal subependyma of the lateral ventricle produce progeny that take on distinct fates in the olfactory bulb and striatum. Compared with the progeny of endogenous striatal stem cells, the cortex-derived (Emx1-lineage GFPpositive) striatal stem cells: (i) proliferate faster in the adult striatal subependyma; (ii) reach the olfactory bulb earlier; (iii) form equivalent proportions of granule neurons but a greater proportion of periglomerular neurons in the olfactory bulb; (iv) form more dopaminergic neurons in the periglomerular layer of the olfactory bulb and (v) form more astrocytes and fewer neurons in the adult striatum. Thus, history matters. However, what element or event in the embryonic history of these cells matters in order to produce differences in their progeny, despite their identical niches within the post-natal striatal subependyma? Does the 'memory' of a neural stem cell depend on gene expression differences induced in the embryonic cortex vs. striatum (and thus the separate niches where they were created), on the migratory journey taken by the cortex-derived neural stem cells but not the endogenous striatal stem cells, on the different total times spent in the striatal germinal zone or on all of these variables?

With respect to gene expression differences induced in the embryonic cortical vs. striatal niches of the two neural stem cell

FIG. 3. Emx1-lineage GFP-positive cells give rise to more cells in the glomerular layer than GFP-negative cells and differentiate in different subtypes of neurons in the granular and glomerular layers of the olfactory bulb. (A) GFP-positive cells (green) from the lateral ventricle in the olfactory bulb. Olfactory bulb cryosections from adult Emx 1-Z/EG mice injected with BrdU (red). Scale bar, $200 \mu \mathrm{m}$. GL, glomerular layer; GR, granular layer; MI, mitral cell layer. (B-E) GFP-positive cells in the mitral cell layer are not mitral cells. Olfactory bulb cryosections from post-natal day 5 Emx1-Z/EG mice. These micrographs represent Tbx21 (B and E, red), GFP (C and E, green) and DAPI (D and E, blue). Scale bar, $50 \mu \mathrm{m}$. (F) Emx1-lineage GFP-positive cells give rise to more cells in the glomerular layer of the olfactory bulb. Adult Emx1-Z/EG mice were injected with BrdU every 3 h over $12 \mathrm{~h}$ and were killed after 6 or 29 days. BrdU-positive and GFP-positive cells were counted in the granular and glomerular layers of the olfactory bulb. The data represent $n=4$ independent experiments; 606 cells were counted at day 6 and 1302 cells at day 29. Error bars represent the SEM. (G-AD) Olfactory bulb cryosections from adult Emx1-Z/EG mice. These micrographs represent cells in the granular layer $(\mathrm{G}-\mathrm{O})$ and glomerular layer $(\mathrm{P}-\mathrm{AD})$ immunolabelled with NeuN (G, I, P and R, red), GAD65 (J, L, S and U, red), calretinin (M, O, V and X, red) tyrosine hydroxylase (TH) (Y and AA, red), Pax6 (AB and AD, red) and GFP (H, I, K, L, N, O, Q, R, T, U, W, X, Z, AA, AC and AD, green). NeuN and GAD65 pictures $(\mathrm{G}-\mathrm{L}$ and $\mathrm{P}-\mathrm{U})$ are confocal images. Confocal microscopic analysis confirmed that these single cells were indeed double-labelled in $>90 \%$ of cases. Scale bar, $25 \mu \mathrm{m}$. 

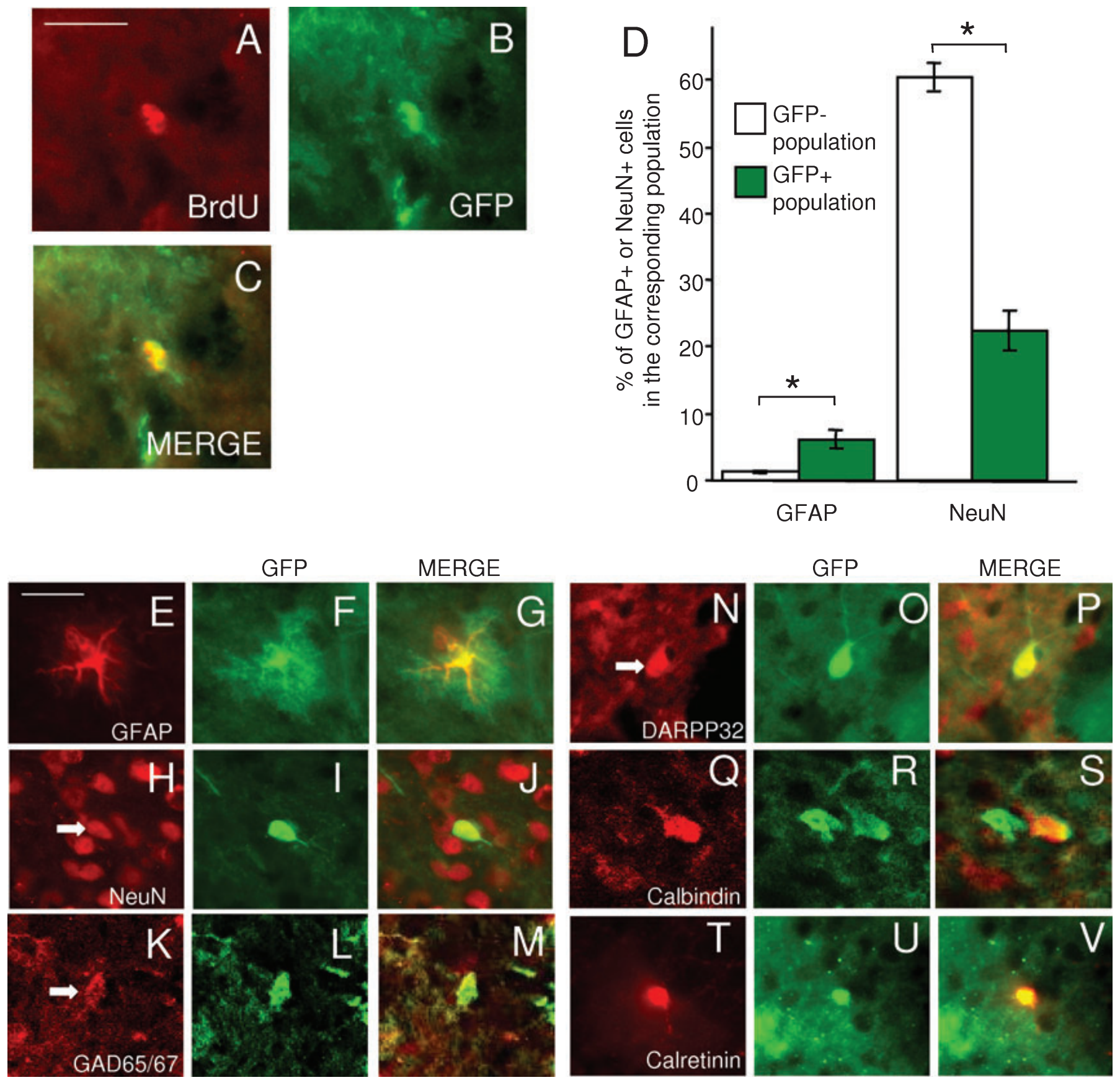

FIG. 4. Emx1-Lineage GFP-positive cells migrate into the striatum and give rise to astrocytes and neurons. (A-C) GFP-positive cells migrate from the lateral ventricle to the striatum. Striatum cryosections from adult Emx1-Z/EG mice injected with BrdU for a total of five injections every $3 \mathrm{~h}$ and killed after 6 days. This micrograph represents BrdU (A and C, red) and GFP (B and C, green). Scale bar, $25 \mu \mathrm{m}$. (D) Emx1-lineage GFP-positive cells give rise to more astrocytes and fewer neurons than GFP-negative cells. Percentages of NeuN-positive and glial fibrillary acidic protein (GFAP)-positive cells in the corresponding populations. The data represent $n=3$ independent experiments; 14515 cells were counted. Error bars represent the SEM. $* t=2.779, P=0.027$ for GFAP and $t=3.886$, $P=0.006$ for NeuN, with a Student's $t$-test. (E-V) Emx1-lineage GFP-positive cells differentiate in astrocytes and different subtypes of neurons in the striatum. Striatal cryosections from adult Emx1-Z/EG mice. These micrographs represent cells in the striatum immunolabelled with GFAP (E and G, red), NeuN (H and J, red), GAD65/67 (K and M, red), DARPP32 (N and P, red), calbindin (Q and S, red), calretinin ( $\mathrm{T}$ and V, red) and GFP (F, G, I, J, L, M, O, P, R, S, U and V, green). GAD65/67 and calbindin pictures (K-M and Q-S) are confocal images. Confocal microscopic analysis confirmed that these single cells were indeed double-labelled in $>90 \%$ of cases. Scale bar, $25 \mu \mathrm{m}$.

types, we have shown previously that cortex-derived neural stem cells maintain their cortical expression of Emx1 for a short perinatal period while in the dorsal striatal germinal zone. However, Emxl is turned off later in these cortex-derived neural stem cells in the post-natal mouse striatal subependyma, where the cortex-derived neural stem cells begin to express Dlx2 like the endogenous striatal neural stem cells (Willaime-Morawek et al., 2006). Thus, the continued expression of Emx1 does not mediate the historical memory of the cortex-derived neural stem cells in the striatal subependyma. Nevertheless, the cortexderived neural stem cells in the striatal subependyma may maintain the 
expression of other dorsal markers, such as Emx2, which is also known to be expressed in many striatal stem cells (Galli et al., 2002). Moreover, Emx 2 plays a role in the proliferation of neural stem cells and cortical precursors (Heins et al., 2001; Galli et al., 2002) and thus a maintained high level of Emx2 expression in cortex-derived neural stem cells and the adult progenitors that they give rise to in the striatal subependyma might also explain the proliferation difference seen in the subependyma between the cortex-derived and endogenous striatal precursor populations.

Another dorsal telencephalic marker that might be differentially expressed within the cortex-derived and endogenous striatal neural stem cells in the striatal subependyma would be Pax6. Indeed, Pax6 is expressed in the embryonic cortex (Stoykova \& Gruss, 1994) and Pax6 directs differentiation towards olfactory bulb periglomerular dopaminergic neurons (Hack et al., 2005) and is required for making a subset of olfactory bulb periglomerular neurons (Vitalis et al., 2000; Kohwi et al., 2005). The present results show that some of the Emx1lineage GFP-positive cells in the glomerular layer of the olfactory bulb express Pax6, with GFP-positive cells three times more likely to become Pax6-positive cells in the glomerular layer $(24.1 \pm 5.0 \%$ of the GFP-positive cells are Pax6-positive) compared with the GFPnegative cells $(8.0 \pm 1.5 \%$ of the GFP-negative cells are Pax6positive). A persistent expression of Pax6 by cortex-derived neural stem cells in the striatal subependyma might explain their tendency to produce more periglomerular and/or dopaminergic neuronal progeny in the olfactory bulb than are produced by the endogenous striatal stem cells in the adult subependyma. Moreover, Pax6 is required for proper proliferation of hippocampus progenitors (Maekawa et al., 2005) and could also contribute to the faster cell cycle times shown here for the cortex-derived precursors compared with host striatal precursors in the subependyma.

The migration history of the cortex-derived subependymal neural stem cell might also contribute to the memory and different fates of the progeny of these stem cells. For example, Emx1-lineage GFP-positive cells that have migrated during the perinatal period from the cortex to the striatal germinal zone are clearly cells with a potent ability for migration. Indeed, the current study shows cortexderived cells reaching the olfactory bulb earlier than striatumderived cells. Given that these cells are already on the move from the cortex to the striatum, these cortex-derived cells and their progeny may also be faster to join the chain migration in the RMS to the olfactory bulb.

Finally, the post-natal endogenous striatal stem cells have clearly spent a longer total time in the striatal subependyma than the neural stem cells that migrate there from the cortex during the perinatal period. Indeed, the cortex-derived GFP-positive neural stem cells arrive in the striatal subependyma at a perinatal time when neurogenesis is ending and gliogenesis is increasing (Sun et al., 2003). Thus, the endogenous striatal neural stem cells have been under the influence of both early neurogenic and later gliogenic environments in the striatal subependyma, whereas the migrating cortex-derived neural stem cells have seen primarily a gliogenic environment in the striatal subependyma. It would be interesting to know whether the two cell populations described here correspond to the two populations of nestin-GFP-bright cells expressing glial fibrillary acidic protein and nestin-GFP-dim cells expressing beta-III-tubulin (Mignone et al., 2004). This different niche history may explain the predominance of glial progeny in the striatum from the cortex-derived neural stem cells in the striatal subependyma.

In conclusion, the history of a stem cell matters for its future. The progeny of cortex-derived striatal neural stem cells have different properties than the progeny of endogenous striatal neural stem cells.

\section{Acknowledgements}

We thank C. Lobe for the gift of Z/EG mice, K.R. Jones for the gift of EmxIRES-Cre mice, Y. Yoshihara for the gift of the Tbx21 antibody, T. Inoue for generating the Ds-red lentivirus and members of our laboratory, in particular R.M. Seaberg, for helpful discussions. This study was supported by the Canadian Stem Cell Network (SWM) and the Canadian Institutes of Health Research. S.W.-M. performed the research and S.W.-M. and D.v.d.K. designed the research, analysed the data and wrote the paper.

\section{Abbreviations}

BrdU, bromodeoxyuridine; GFP, green fluorescent protein; RMS, rostral migratory stream; Z/EG, lacZ/enhanced green fluorescent protein.

\section{References}

Chan, C.H., Godinho, L.N., Thomaidou, D., Tan, S.S., Gulisano, M. \& Parnavelas, J.G. (2001) Emx1 is a marker for pyramidal neurons of the cerebral cortex. Cereb. Cortex, 11, 1191-1198.

Davis, A.A. \& Temple, S. (1994) A self-renewing multipotential stem cell in embryonic rat cerebral cortex. Nature, 372, 263-266.

Galli, R., De Fiocco, R.F.L., Muzio, L., Gritti, A., Mercurio, S., Broccoli, V., Pellegrini, M., Mallamaci, A. \& Vescovi, A.L. (2002) Emx2 regulates the proliferation of stem cells of the adult mammalian central nervous system. Development, 129, 1633-1644.

Gorski, J.A., Talley, T., Qiu, M., Puelles, L., Rubenstein, J.L. \& Jones, K.R. (2002) Cortical excitatory neurons and glia, but not GABAergic neurons, are produced in the Emx1-expressing lineage. J. Neurosci., 22, 6309-6314.

Hack, M.A., Saghatelyan, A., de Chevigny, A., Pfeifer, A., Shery-Padan, R., Lledo, P.M. \& Gotz, M. (2005) Neuronal fate determinants of adult olfactory bulb neurogenesis. Nat. Neurosci., 8, 865-872.

Heins, N., Cremisi, F., Malatesta, P., Gangemi, R.M., Corte, G., Price, J., Goudreau, G., Gruss, P. \& Gotz, M. (2001) Emx2 promotes symmetric cell divisions and a multipotential fate in precursors from the cerebral cortex. Mol. Cell Neurosci., 18, 485-502.

Kohwi, M., Osumi, N., Rubenstein, J.L. \& Alvarez-Buylla, A. (2005) Pax6 is required for making specific subpopulations of granule and periglomerular neurons in the olfactory bulb. J. Neurosci., 25, 6997-7003.

Kohwi, M., Petryniak, M.A., Long, J.E., Ekker, M., Obata, K., Yanagawa, Y., Rubenstein, J.L. \& varez-Buylla, A. (2007) A subpopulation of olfactory bulb GABAergic interneurons is derived from Emx1- and Dlx5/6-expressing progenitors. J. Neurosci., 27, 6878-6891.

Kornack, D.R. \& Rakic, P. (2001) Cell proliferation without neurogenesis in adult primate neocortex. Science, 294, 2127-2130.

Laywell, E.D., Rakic, P., Kukekov, V.G., Holland, E.C. \& Steindler, D.A. (2000) Identification of a multipotent astrocytic stem cell in the immature and adult mouse brain. Proc. Natl Acad. Sci. U.S.A., 97, 13 883-13 888 .

Luskin, M.B. (1993) Restricted proliferation and migration of postnatally generated neurons derived from the forebrain subventricular zone. Neuron, 11, 173-189.

Maekawa, M., Takashima, N., Arai, Y., Nomura, T., Inokuchi, K., Yuasa, S. \& Osumi, N. (2005) Pax6 is required for production and maintenance of progenitor cells in postnatal hippocampal neurogenesis. Genes Cells, 10, 1001-1014.

Merkle, F.T., Mirzadeh, Z. \& varez-Buylla, A. (2007) Mosaic organization of neural stem cells in the adult brain. Science, 317, 381-384.

Mignone, J.L., Kukekov, V., Chiang, A.S., Steindler, D. \& Enikolopov, G. (2004) Neural stem and progenitor cells in nestin-GFP transgenic mice J. Comp. Neurol., 469, 311-324.

Morshead, C.M. \& van der Kooy, D. (1992) Postmitotic death is the fate of constitutively proliferating cells in the subependymal layer of the adult mouse brain. J. Neurosci., 12, 249-256.

Novak, A., Guo, C., Yang, W., Nagy, A. \& Lobe, C.G. (2000) Z/EG, a double reporter mouse line that expresses enhanced green fluorescent protein upon Cre-mediated excision. Genesis, 28, 147-155.

Seaberg, R.M., Smukler, S.R. \& van der Kooy, D. (2005) Intrinsic differences distinguish transiently neurogenic progenitors from neural stem cells in the early postnatal brain. Dev. Biol., 278, 71-85.

Simeone, A., Gulisano, M., Acampora, D., Stornaiuolo, A., Rambaldi, M. \& Boncinelli, E. (1992) Two vertebrate homeobox genes related to the 
Drosophila empty spiracles gene are expressed in the embryonic cerebral cortex. EMBO J., 11, 2541-2550.

Stoykova, A. \& Gruss, P. (1994) Roles of Pax-genes in developing and adult brain as suggested by expression patterns. J. Neurosci., 14, 13951412.

Sun, Y.E., Martinowich, K. \& Ge, W. (2003) Making and repairing the mammalian brain - signaling toward neurogenesis and gliogenesis. Semin. Cell Dev. Biol., 14, 161-168.

Ventura, R.E. \& Goldman, J.E. (2007) Dorsal radial glia generate olfactory bulb interneurons in the postnatal murine brain. J. Neurosci., 27, 42974302 .
Vitalis, T., Cases, O., Engelkamp, D., Verney, C. \& Price, D.J. (2000) Defect of tyrosine hydroxylase-immunoreactive neurons in the brains of mice lacking the transcription factor Pax6. J. Neurosci., 20, 6501-6516.

Willaime-Morawek, S., Seaberg, R.M., Batista, C., Labbe, E., Attisano, L., Gorski, J.A., Jones, K.R., Kam, A., Morshead, C.M. \& van der Kooy, D. (2006) Embryonic cortical neural stem cells migrate ventrally and persist as postnatal striatal stem cells. J. Cell Biol., 175, 159-168.

Young, K.M., Fogarty, M., Kessaris, N. \& Richardson, W.D. (2007) Subventricular zone stem cells are heterogeneous with respect to their embryonic origins and neurogenic fates in the adult olfactory bulb. J. Neurosci., 27, $8286-8296$. 